\section{PARKINSON DISEASE}

\section{Long-term levodopa exposure may increase risk of neuropathy in patients with PD}

Peripheral neuropathies are thought to be prevalent among patients with Parkinson disease (PD), and might contribute substantially to motor disability in this population. In a new study reported in Movement Disorders, Roberto Ceravolo, Ubaldo Bonuccelli and colleagues have identified age and long-term levodopa treatment as independent risk factors for neuropathy in patients with PD.

According to Ceravolo, previous investigations into the link between neuropathy and levodopa exposure in PD have been limited by small sample sizes, inadequate electrophysiological assessment, and lack of a levodopa-naive PD control group. "With these issues in mind, we designed a large multicentre study to investigate-both clinically and electrophysiologically_-PD patients with long or short durations of exposure to levodopa, patients never treated with levodopa, and a group of matched healthy controls," he explains.
Ceravolo et al. recruited patients from six tertiary referral centres for PD across Italy. Their study included 144 PD patients with long-term ( $>3$ years') levodopa exposure, 103 PD patients with short-term ( $<3$ years') levodopa exposure, $83 \mathrm{PD}$ patients with no levodopa exposure, and 137 healthy controls. The reduced version of the Total Neuropathy Score was used to quantify nerve function.

Ceravolo et al. found that $19.40 \%$ of the patients with long-term levodopa exposure exhibited neuropathy, compared with only $6.80 \%$ of those with shortterm exposure, $4.82 \%$ of those with no exposure, and $8.76 \%$ of the controls. The risk of neuropathy also increased with age, but was not influenced by disease duration or severity.

The researchers found that patients with neuropathy had, on average, higher plasma homocysteine levels and lower plasma vitamin $\mathrm{B}_{12}$ levels than

\section{$44 . . .19 .40 \%$ of the patients with long-term levodopa exposure exhibited neuropathy... $\$ 7$}

did patients without neuropathy. These findings suggest possible roles for catechol-O-methyltransferase inhibition-which reduces plasma homocysteine levels - and vitamin $\mathrm{B}_{12}$ supplementation as preventative measures for neuropathy in patients with PD.

"We should be aware that, in the setting of advanced PD and a long history of levodopa treatment, the prevalence of neuropathy is significant and of clinical relevance," concludes Ceravolo. "The practical implications of our observations include the need for periodic peripheral nerve assessment during long-term levodopa treatment."

\section{Heather Wood}

Original article Ceravolo, R. et al. Neuropathy and levodopa in Parkinson's disease: evidence from a multicenter study. Mov. Disord. doi:10.1002/mds.25585 